\title{
Resilient design of distribution grid automation system with CALVIN
}

\author{
Abhinav Sadu,Lukas Ostendorf,Gianluca Lipari, Ferdinanda Ponci, Antonello Monti \\ Institute for Automation of Complex Power Systems, E.ON Energy Research Center, RWTH University \\ Aachen, Germany[asadu, lostendorf,glipari,fponci,amonti]@eonerc.rwth-aachen.de
}

\begin{abstract}
The distribution automation system is vital for the safe and reliable operation of distribution grids. Typically, the automation architectures are either centralized or hierarchical. The monitoring and controlling of the grid is done by dedicated actors like the Supervisory Control And Data Acquisition (SCADA) systems and the Distribution Management Systems (DMS) or the Substation Automation Units (SAUs). These actors are deployed in dedicated machines and thus become a single point of failure in the automation system. Hence, the chances of blackouts are increased when any of these dedicated machines are compromised (due to either cyber-attacks or natural calamities). Therefore, special measures have to be taken that enable the migration of the functionalities of SCADA/DMS/SAU to a clean new machine, when the previous machine is compromised. This ensures higher availability and resilience of grid monitoring and control applications. This study presents the feasibility of using CALVIN IoT framework for providing such resiliency. A basic architecture for enabling the migration strategies with CALVIN is proposed and implemented. The results show that the CALVIN framework is a promising solution in providing resilience to the distribution grid automation.
\end{abstract}

Keywords-CALVIN, Resilience, Substation automation

\section{INTRODUCTION}

The deregulation of the energy markets and the increasing penetration of Distribution Energy Resources (DERs), calls for deployment of Advance Distribution Automation (ADA) systems, which ensure adequate quality of supply of the distribution grids. The ADA system generally includes the sensors, measurement devices, actuators, measurement units, control units and finally the communication infrastructure for the data exchange between the aforementioned actors

Traditionally the distribution automation systems have a centralized architecture. Generally the data is collected at a central location from the different field devices like the measurement units and the Remote Terminal Units (RTUs) by the Supervisory Control And Data Acquisition System [1]. The collected data is processed by Distribution grid Management System (DMS), which hosts the different monitoring and control applications like the State Estimation (SE), Volt/Var control, network congestion management and so on. These applications ensure the safety and reliability of the distribution grids. However, both the SCADA and the DMS are generally deployed in dedicated servers in a control centre. Any attack on

This project has received funding from the European Union's Horizon 2020 research and innovation program under grant agreement No 700416 these servers resulting in their failure would cause the loss of the operational capabilities with possible consequent blackout. The coordinated cyber-attacks performed on the ADA system of the Ukranian DSO exploited this vulnerability [2]. The SCADA/DMS system was hijacked, critical monitoring \& control algorithms were erased, wrong control commands were sent, which triggered unwanted outages and thus resulting in a blackout lasting several hours [2].

In order to improve the performance of the ADA system, a decentralized automation architecture has been proposed within the IDE4L project [3][4]. In the proposed architecture, the intelligence for grid operation was allocated to individual MV and LV substations for managing their respective downstream grid, using the Substation Automation Unit (SAU). This reduces the computational and communication burden to the SCADA/DMS. Furthermore, each SAU is responsible for operating a specific segment of the grid and interacts with the control center and other SAUs for coordinated control of the whole distribution grid. Such a de-centralized automation architecture is realizable and scalable because of the advances in the Information \& Communication Technology (ICT) infrastructure. Specifically due to the IP based Wide Area Communication and standardized data modelling schemes. Unlike the Centralised Architecture (CA), the failure of a single SAU results in the failure of only a segment of the grid and not the whole grid. Nevertheless, the SAU is equally vulnerable as the SCADA/DMS in CA, as it is also deployed on dedicated computational hardware.

The SCADA/DMS of the Ukranian DSO was compromised with different coordinated attacks that included the phishing attacks, credential theft, distributed denial-of-service (DDoS) attack, killDisk attack and unauthorized VPN/Remote Terminal access [2]. Several counter measures are proposed in the literature against these attacks [5][6]. However, these countermeasures protect the SCADA/DMS/SAU from potential cyber-threat, they don't provide mechanisms that ensures availability of their functions once they have been compromised. This study adopts the automation architecture proposed in IDE4L project and the authors propose a method that virtualizes the functions of the SAU and migrates them to a clean hardware, as soon as the machine that was previously hosting it, is compromised. This ensures the availability of the SAU and increases the resilience of the automation system. In this work, the authors investigate the capability of the CALVIN 
IoT framework to virtualize the functionalities of SAU. The performance of CALVIN concerning the achievable data rates, the extent of distribution of automation functionalities and latencies involved in the migration process are presented. Finally the performance of the different migration strategies possible with CALVIN is evaluated.

\section{DISTRIBUTION GRID AUTOMATION : IDE4L}

The IDE4L automation architecture for distribution grid is decentralized and modular and it is specified in terms of interfaces, database and grid monitoring \& control applications [7]. One of the major actors in the IDE4L architecture is the SAU, which is responsible for the monitoring and control of individual segments of the distribution grid, as shown in an example in Fig.1. The SAU consists of three major components, namely the interfacing layer, database and the application layer. The interfacing layer is primarily a communication protocol translator responsible for interacting with the field devices. The data corresponding to the real-time measurements, statuses of protection devices, control set points to be sent to remote control units are all stored in the database. The different monitoring and control applications are namely, the state estimation, voltage \& frequency control applications. These form the application layer that interacts with the database for updated measurements and statuses.

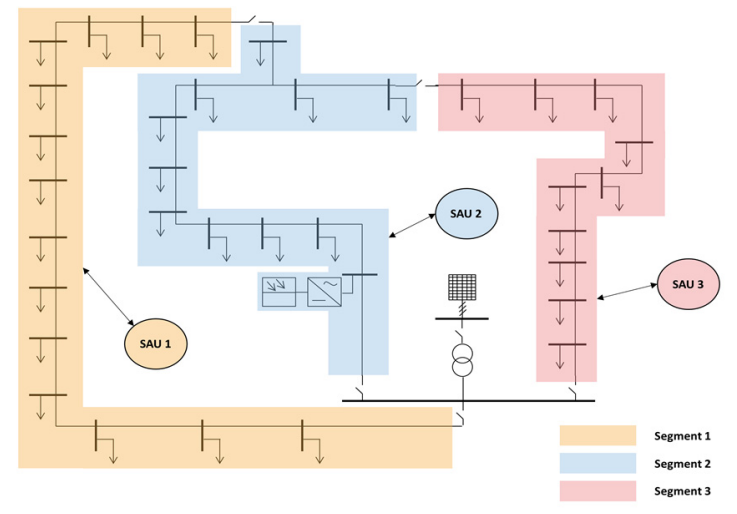

Fig.1. Distribution grid automation with SAUs : an Overview

By virtualizing the SAUs it is possible, in case of an attack targeting one of the instances, to quickly migrate or reinitiate such instance on a new device, while the other instances can continue to run unaffected by such incident. This allows enhancing the resilience of the single instance. For this purpose, in this study we adopted the CALVIN Framework.

\section{CALVIN FRAMEWORK}

CALVIN is a distributed IoT framework made available as open source package by Ericsson. It combines the idea of the Actor model and flow based programming [8]. CALVIN provides a simplified framework which eases the development of the application for distributed systems. The framework consists of the three-architecture layers including runtime, actors and an application.

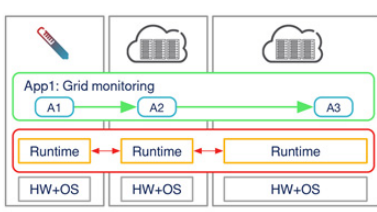

(a)

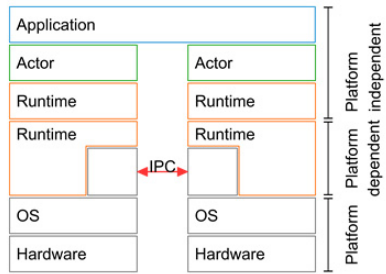

(b)
Fig.2. (a) The distributed execution of the application; (b) The software stack of CALVIN[9]

As shown in the Fig.2, an application in the CALVIN framework can be implemented as combination of the actor. Where each actor is responsible for the part of the logic. In fact, opposite to sequential programming models, in CALVIN the programmer does not have to explicitly create processes that can be executed in parallel. Instead, the actions of actors will automatically be executed in parallel, wherever the implemented algorithms allow it [9]. This is the main advantage of implementing application as set of actors in CALVIN. The runtime shown in the Fig.2, sits on the top of the Operating System. The runtime includes protocols over which actors can communicate and also it abstracts the platform independent functionalities. In addition, each runtime includes a scheduler, which coordinates the execution of the actors running on top of it. Furthermore, each runtime has a REST-compliant API, over which users can control the runtime and the applications, including the creation of a new application or a single actor and the migration of actors.

The important feature, relevant for resilient design of the distributed automation application, is the flexible allocation and deployment of the actors. Multiple runtime nodes connected together form a meshed network, in which actors can be migrated between different runtimes. The speed of migration depends upon the speed of transport between devices and the computational power of the devices. This allows seamless migration of distribution grid automation functions from one runtime to another. The migration of the actors by CALVIN is done without interrupting the execution of the grid automation function. For which security measures to authorize the access is done using the fine grain attribute based access authorization is included in the framework[10]. Information on the actors can be stored in a distributed manner within the network. This is implemented by the distributed hash-table Kademlia [11]. Distributed storage increases the stability of the system with respect to the central storage, since it avoids single point of failure. The DHT is strengthened with node identification mechanisms, cryptographic message exchange schemes and certificate distributoion schemes. A detailed description of these schemes and their performance against specific cyber security threats have been presented in [12].

In this work, the authors have realized distributed automation functions as actors in the CALVIN framework. Further, Section $\mathrm{V}$ presents investigation on the performance of CALVIN in terms of the migration of the distribution grid automation functions in a mesh network of the run-times. Each acting as a complete SAU or its segments. 
IV.

TeSt CASE IMPLEMENTATION

The test case includes three different hardware where CALVIN environment is run. Each hardware corresponds to a SAU. Each SAU hosts a specific application. This case study is a basic implementation of a real time monitoring of a distribution grid, where a state estimation algorithm runs in a SAU that receives real time measurements data from other SAUs, using the CLAVIN framework .For this the different components of the SAU as described in Section II are realized as individual CALVIN actors, within the CALVIN framework. The different actors are the individual monitoring application (SE), and the instance of TCP server/client, representing the application and interfacing layer of SAU respectively. The test lab setup for the performance evaluation of CALVIN and the migration strategies is presented in the following sections.

\section{A. $\quad$ Test lab setup}

The purpose of this lab setup is to test the performance of the CALVIN framework for the migration of SAU. This is indicated by the performance metrics like latency for migration, maximum data rates possible between actors, and latencies of data exchange between actors.

\section{1) Hardware setup}

The hardware setup consists of two Raspberry Pi 3 and a server running Linux, the configuration is as listed in Table I . They are connected through Ethernet. All devices are running a CALVIN runtime, the server additionally hosts an IED App which simulates a measurement device and control unit. Furthermore it hosts the Remote Actor Manager that manages the different actors in different runtimes. This system setup is shown in Fig.3.

TABLE I. SPECIFICATIONS OF THE USED HARDWARE

\begin{tabular}{|c|c|c|}
\hline \multirow{2}{*}{ Component } & \multicolumn{2}{|c|}{ Computing Machines } \\
\cline { 2 - 3 } & Linux Server & Raspberry PI 3 \\
\hline CPU & $2 \times 2.7 \mathrm{GHz}$ & $4 \times 1.2 \mathrm{GHz}$ \\
\hline RAM & $8 \mathrm{~GB}$ DDR3 & $1 \mathrm{~GB}$ \\
\hline OS & Ubuntu 16.04 & Raspian 4.9 \\
\hline CALVIN & Version 0.7 & Version 0.7 \\
\hline Networking & $100 \mathrm{Mbit}$ LAN & $100 \mathrm{Mbit}$ LAN \\
\hline
\end{tabular}

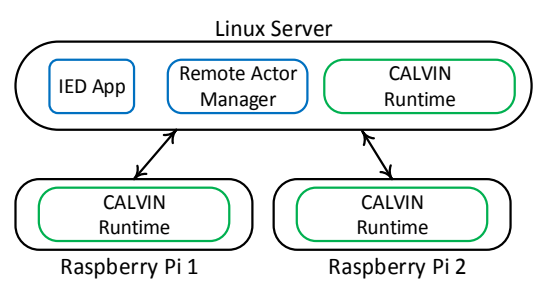

Fig.3. System Setup

\section{2) Actor migration strategies}

In this study we assume that the attack detection schemes are already in place, which sense an attack and trigger the Remote Actor manager to initiate the migration process. To migrate an actor, The Remote Actor Manager sends a request to the API of the runtime, where the actor is currently running. In this request, the unique ID of the actor instance and the ID of the destination runtime is specified. The migration process starts by letting the actor execute a function to do an environment cleanup, such as closing open TCP connections or stopping timers. Next, the connections to other actors are disconnected. After that, the actual migration process starts. The actor is deleted on the old runtime and migrated to the new runtime. Since the logic of the actor is already available at each runtime, the actual program code of the actor is not transmitted. Instead, only the type of the actor and the current actor state are transmitted. When the destination runtime receives the data, it creates a new actor and restore the state. The ports are reconnected and finally an initialization function of the actor is called. After the successful actor creation, the destination runtime acknowledges this to the source runtime, which then acknowledges the success to the Remote Actor Manager. The two migration strategies possible with CALVIN are described below:

a) Restarting the complete environment : This migration procedure is adopted when the CALVIN runtime is not under the control of the Remote Actor Manager anymore. This may occur due to DDOS attacks on the hardware that hosts SAU or when it is physically damaged. In this case, we have to re-create the actors which were running on the affected device. After recreating the actors, the actor ports have to be reconnected to the rest of the application. The reconnecting of the ports is supported by the REST-API of the runtime. However, since the old port connections from the faulty actor are not closed properly, disconnecting them would not work. Therefore restarting individual actors is not possible. Instead, we have to re-create all the actors of the attacked environment.

b) Migrating the actors: This migration procedure is adopted when a device is currently attacked, but is still manageable. In this scenario, the Remote Actor Manager sends commands to the CALVIN runtime using the CALVIN migration feature. The migration command must specify the actor which shall be migrated and its destination runtime. All actors, which are currently being executed on the attacked runtime, are migrated to a non-attacked destination runtime.

\section{3) Configuration of CALVIN runtimes}

The configuration of the CALVIN environments on the different hardware is as shown in Fig.4. The functions of the different actors is as follows

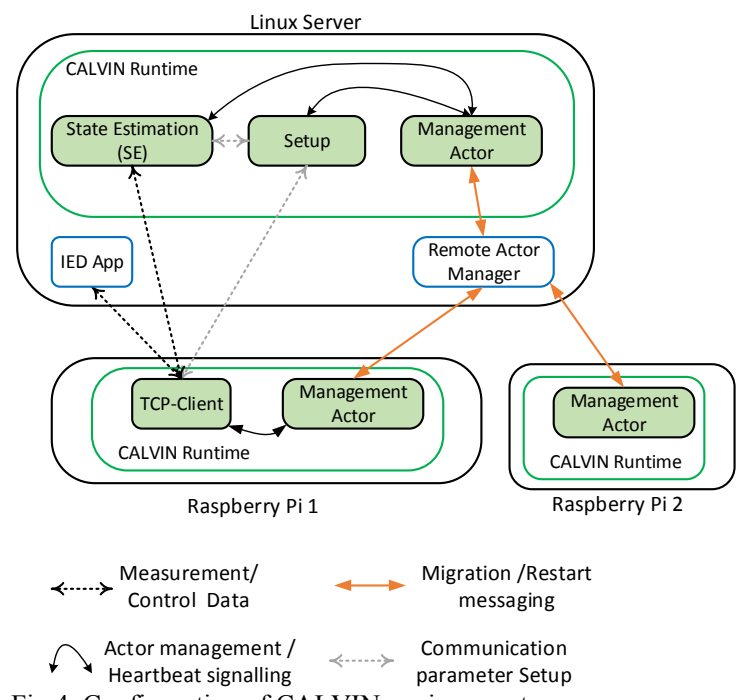

Fig.4. Configuration of CALVIN environments 
- Management Actor : This actor is responsible for detecting live actors within the CALVIN runtime where it is deployed. A heartbeat technique is used, where the management actor sends periodic commands to all the other actors deployed in the runtime, to which, each connected actor acknowledges back with an ACK message. The Management actor then informs the Remote Actor Manager of the disconnected and the connected actors. Additionally, all actors can send a disconnect message to the management actor. This message is sent when an actor is shut down properly or if the actor is going to migrate. The feature is needed to prevent false positives, since a migrating actor is not available for a short time and therefore might not be able to answer a heartbeat signal. The heartbeat signal is a JSON message, to comply with CALVIN's requirements on token types. The message contains the actor ID and their status like "connected" if it is connected and "disconnected" if the actor is about to migrate.

- $\quad$ TCP-Client : Represents the data interfacing layer of SAU. Responsible for acquiring measurements and send control set points from and to remote IEDs respectively.

- Grid Monitoring Actor \& Grid Control Actor : These actors represent the different monitoring and control applications that run in SAU that are responsible for operating the grid in real time. For this study it is a State Estimation function (SE)

- Setup : This actor is needed for the initial TCP connection configuration

\section{B. Implementation of the grid monitoring application with CALVIN}

A specific type of monitoring application "State Estimator" actor is implemented in CALVIN. The State Estimator actor implements the Weighted Least Square (WLS) Method based power grid state estimation. For simplicity, the necessary information on the grid structure is stored in the code of the actor. It would be possible to separate this into another actor, which fetches the information from a database. The TCP-client actor simulates the communication interface of the SAU that is responsible to connect with remote IEDs, acquires measurements, and forwards it to the State Estimation actor. To emulate the remote IED a TCP server ( IED App) has been configured outside the CALVIN environment to which the TCP-client connects and requests for measurements at regular intervals. Dummy measurements are sent as IEEE754 encoded floats. The TCP-client actor decodes the floats and forwards them through a CALVIN port to the state estimator actor. The estimated states are then, written back on the TCP server (IED App) using the TCP-client actor. This emulates the setting of the set points of the IED. If the TCP-client actor of our CALVIN application is migrated to another runtime, the actor closes the TCP connection and tries to connect to the server again after it migrated. To permit a fast reconnection after the migration, the TCP server has to check constantly for new incoming connections. Therefore, the IED App runs two parallel processes. One is waiting for new incoming connections and the other handles sending measurement data and receiving control set point data. The Remote Actor Manager is responsible for migrating/restarting of the different actors from runtime environment to another.

\section{TEST CASES \& RESUlts}

Several tests are conducted to investigate the performance of CALVIN environment, not only to run the grid automation functions under normal operation conditions but also deploy the migration strategies under cyber/physical attack notification. Regarding the general CALVIN framework, the achievable bandwidth of the CALVIN communication ports and the delay introduced by them during data exchange between actors is characterized. Then the performance of the two migration strategies, namely migration of individual actors and restarting of the actors is presented.

\section{A. CALVIN communication port bandwidth characterization}

These tests evaluate the maximum bandwidth that could be supported by the CALVIN ports for communication between the actors both within the same environment and between actors in different environments. The configuration for this test is as shown in Fig.5.

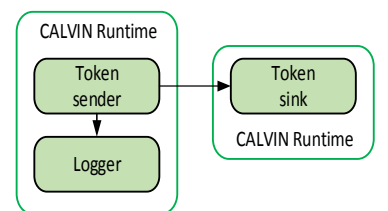

Fig.5. Actor configuration for port bandwidth characterization

CALVIN uses its "local" port communication for communicating between actors in the same environment and uses "CALVIN tunnel" for communicating between actors in different environments. Tests were executed on the Linux server with the hardware configuration shown in Table I. Fig.6 shows the bandwidths for different token sizes.

The maximum transfer rate is roughly 650MByte per second. The local connection communicates by directly writing and reading a python array. Therefore, the data-rate is only limited by the speed of the RAM and the implementation of the copy command on Python and OS level. The data-rates are compared to the data-rates reached with a python copy script, which repeatedly copies data using the python copy.copy() command. The data rates achieved by CALVIN is close to the maximum achievable using the specific hardware. Thus it can be concluded, that using the CALVIN framework does not affect the data-rates, when transferring data between actors on the same device.

Furthermore the bandwidth of the "CALVIN tunnel", enabling communication between actors in different environments, is evaluated. The achievable bandwidth with CALVIN tunnel is compared with the theoretical bandwidth calculated using the equation (1). The theoretical bandwidth is 
calculated considering the semantics of the CALVIN tunnel protocol as explained subsequently.

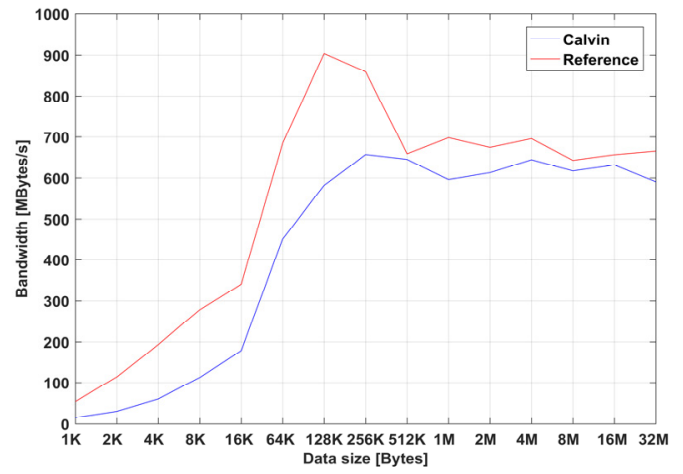

Fig.6. Data-rates for a CALVIN "local" connection

For each token sent, additional control data is attached, including specifications of the source port ID and destination port ID. Furthermore, each message has to be acknowledged, by replying with an ACK. With each token, 434 Bytes control data is sent, the acknowledgement is 416 Bytes long. Based on this information maximum net-bandwidth is calculated. The Fig.7 shows the calculated bandwidth for a $100 \mathrm{MBps}$ connection with $0.3 \mathrm{~ms}$ round-trip time and a 100MBps connection with $22 \mathrm{~ms}$ round-trip time. The actual bandwidth was tested between two Raspberry PIs connected over $100 \mathrm{MBps}$ Ethernet with $0.3 \mathrm{~ms}$ delay. The impact of the delay to the bandwidth, especially when sending small to medium token sizes is observed. Furthermore, the measured bandwidth is significantly lower than the estimated one. It even drops for big token sizes. This is due to the limited computational resources of Raspberry PI. We can conclude that the delay between the actors and the computational resource of the hardware implementing the CALVIN environment, are the deciding factors of the achievable bandwidth.

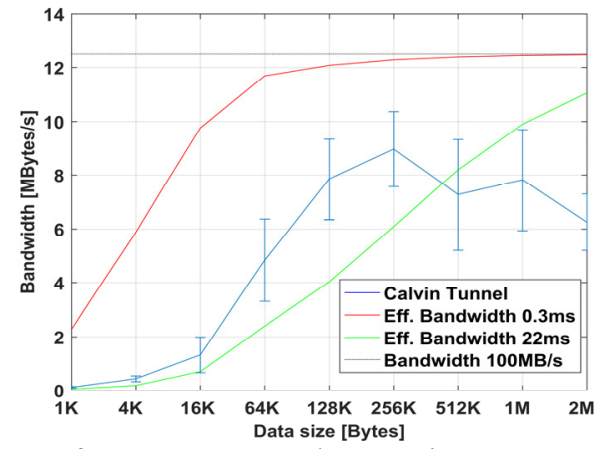

Fig.7. Data-rates for a CALVIN "tunnel" connection

\section{B. CALVIN communication port delay characterization}

This test is performed to determine the delay between two actor ports. The CALVIN environment is configured as shown in Fig. 8 for this test. In theory, the actors react immediately when they receive a token. However, in reality the actor scheduler introduces a certain delay. All actors of this application were deployed on the same runtime, so the measured delays are solely dependent on the delay caused by the scheduler. The results for connections of different hop length are plotted in Fig.9. This test was executed on a Raspberry PI. The delay ranges from $2 \mathrm{~ms}$ for a 1-hop connection to $120 \mathrm{~ms}$ for an 18-hop connection.

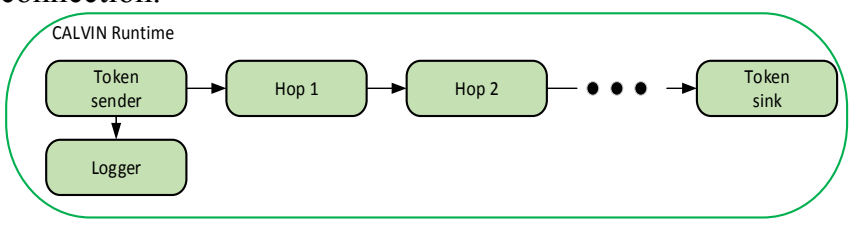

Fig.8. Actor configuration for port delay characterization

Such delays are definitely significant to the computation time, if the program is split into several actors. Furthermore, the overall delay is related to the square of the number of hops. This can be explained with the design of the scheduler function. At the beginning, a list, which contains each active actor of the runtime, is shuffled. Then the scheduler sequentially tries to fire all actions of each actor.

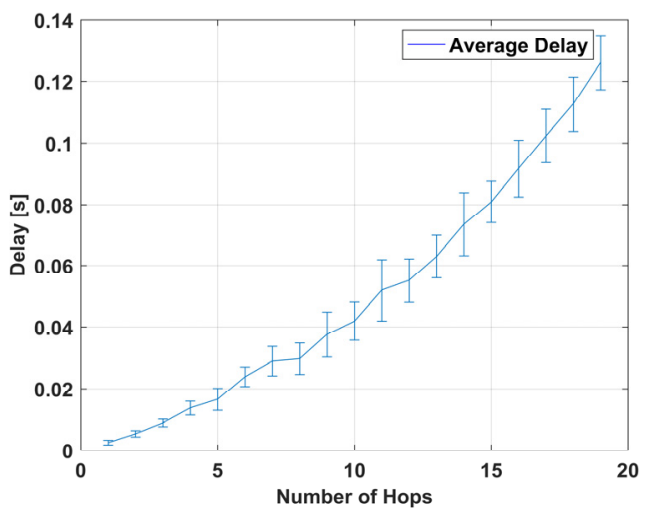

Fig.9.Delay for a connection of different hop length ( $\mathrm{n}=120$ measurements for each hop number)

Fig.10 shows the delay per hop for different numbers of executed actors. The delay per hop increases linearly with the number of active actors. We can conclude from this test, that the delay of a port connection is significant for the computation time of a distributed program. The delay also increases with the number of actors executed on a runtime. This must be considered when choosing the grade of distribution of a monitoring and control application over different actors.

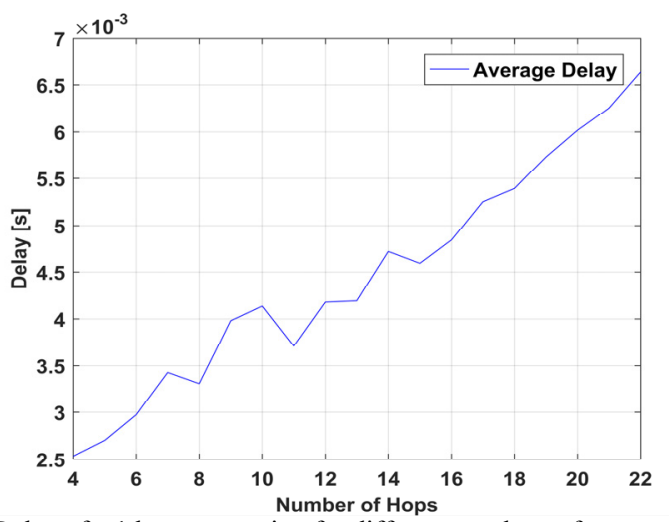

Fig.10. Delay of a 1 hop connection for different numbers of executed actors 
C. Performance of actor migration/restart for power grid automation resiliency

The performance of the CALVIN framework in terms of the total delay in either migrating the actors or restarting the actors is presented in this section, after carefully considering the tradeoffs pertaining to the achievable bandwidth and delay of the CALVIN communication ports.

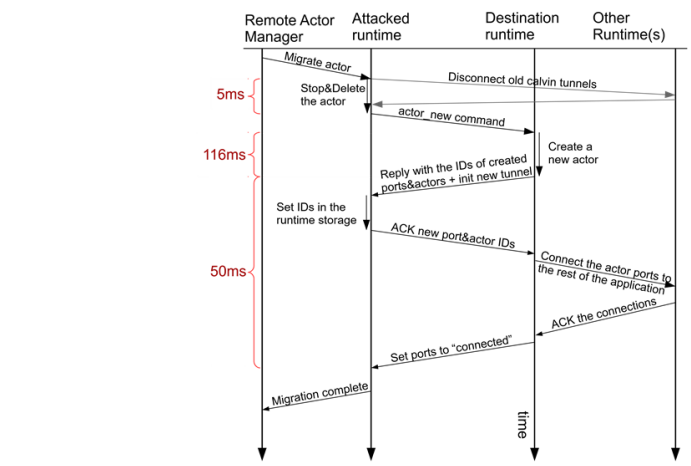

Fig.11. Delay characterization of actor migration

Fig.12. Delay characterization of actor restart

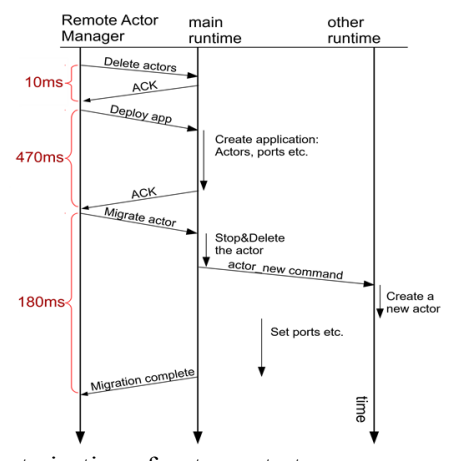

Migration is performed when the specific attacked node is alive and is responsive to the rest of the network. The restarting of actors is performed when a specific CALVIN node is either not reachable due to physical damages or due to cyber-attacks. For this test the CALVIN environments have been configured as depicted in Fig.4. The migration of the SE actor into the Raspberry Pi 2 is performed. The results are as shown in Fig. 11 \& Fig. 12 respectively for the migration and restarting of actors. In Fig. 11 the Attacked runtime depicts the targeted runtime whose actors need to be migrated. The migration takes less time than the restarting of the actors. This is attributed to the amount of interactions between the attacked runtime (actors of which need to be migrated) and the destination runtimes while migrating the actors.

In practice the complete SCADA/SAU functions deployed as individual actors could be migrated or restated. Specifically, from one control center to another (for SCADA) or from one substation to another (for SAU). The migration/ restart delays depend heavily on the latencies introduced by the communication infrastructure. Therefore, as future work, optimally selection of nodes for migration, taking into account the reserve computational power, memory storage space and communication latencies has to be made.

\section{CONCLUSIONS \& FUTURE WORK}

In this work, the authors evaluate the performance of the CALVIN framework in improving the availability of distribution grid automation systems. A mapping of the automation functionalities of SAU (as an example in this study), to the actors of CALVIN is proposed. This ensures the virtualization of the functionalities of SAU and thus enabling its migration. From the test results it is clear that, due consideration have to be made in distributing the grid monitoring control and protection application into different actors. From the results (Fig.10), we can conclude that any application that requires a faster reaction time should be distributed in fewer actors. Two migration strategies that could be implemented with CALVIN in ensuring the availability of SAU is presented and evaluated. The results show that both migration strategies implemented with CALVIN provide sub second latencies for the migration. Thus ensuring reduction in the downtime of the automation system under cyber and physical attacks. Of course the latencies introduced by the communication infrastructure would add on top of the presented results. For the future work, the authors would also extend this platform to include the migration of databases and propose a distributed architecture for implementing the migration procedure.

\section{REFERENCES}

[1] F. F. Wu, K. Moslehi and A. Bose, "Power System Control Centers: Past, Present, and Future," in Proceedings of the IEEE, vol. 93, no. 11, pp. 1890-1908, Nov. 2005.

[2] R.M.Lee,M.J.Assante,T.Conway, "Analysis of the Cyber Attack on the Ukrainian Power Grid : Defnse Use Case", Washington, March 2016. Online: https://ics.sans.org/media/E-ISAC_SANS_Ukraine_DUC_5.pdf

[3] IDE4L, deliverable 3.2, "Architecture design and implementation," 2015.[Online]. Available: http://ide4l.eu/results/

[4] A. Angioni et al., "Design and Implementation of a Substation Automation Unit," in IEEE Transactions on Power Delivery, vol. 32, no. 2, pp. 1133-1142, April 2017.

[5] Loukas, G.; Gan, D.; Vuong, T. A Review of Cyber Threats and Defence Approaches in Emergency Management. Future Internet 2013, 5, 205 236.

[6] Gelenbe, E.; Wu, F.-J. Future Research on Cyber-Physical Emergency Management Systems. Future Internet 2013, 5, 336-354.

[7] A. Angioni et al., "A distributed automation architecture for distribution networks, from design to implementation", In Sustainable Energy, Grids and Networks, 2017

[8] Per Persson, Ola Angelsmark, CALVIN- Merging Cloud and IoT, In Procedia Computer Science, Volume 52, 2015, Pages 210-217.

[9] Gul A Agha. "Actors: A model of concurrent computation in distributed systems." PhD thesis. Massachusetts inst of tech Cambridge artificial intelligence lab, 1985.

[10] Nillson Tomas. "Authorization Aspects of the Distributed Datafloworiented IoT Framework Calvin.” Student Paper. Lund University, 2016. Online: http://lup.lub.lu.se/student-papers/record/8879081

[11] Petar Maymounkov and David Mazieres. "Kademlia: A peer-to-peer information system based on the xor metric". In: International Workshop on Peer-to- Peer Systems. Springer. 2002, pp. 53-65

[12] Lindskog, Niklas. "Consistent authentication in disitrbuted networks" Master Thesis. Lund University, 2016. Online: http://lup.lub.lu.se/student-papers/record/8871006. 